\title{
A conserved role of the RSC chromatin remodeler in the establishment of nucleosome-depleted regions
}

\author{
Carlo Yague-Sanz ${ }^{1}$ Enrique Vázquez ${ }^{2} \cdot$ Mar Sánchez $^{2} \cdot$ Francisco Antequera $^{2}$ • \\ Damien Hermand ${ }^{1}$ (i)
}

Received: 7 August 2016 / Accepted: 18 August 2016 / Published online: 24 August 2016

(C) The Author(s) 2016. This article is published with open access at Springerlink.com

\begin{abstract}
The occupancy of nucleosomes governs access to the eukaryotic genomes and results from a combination of biophysical features and the effect of ATP-dependent remodelling complexes. Most promoter regions show a conserved pattern characterized by a nucleosome-depleted region (NDR) flanked by nucleosomal arrays. The conserved RSC remodeler was reported to be critical to establish NDR in vivo in budding yeast but other evidences suggested that this activity may not be conserved in fission yeast. By reanalysing and expanding previously published data, we propose that NDR formation requires, at least partially, RSC in both yeast species. We also discuss the most prominent biological role of RSC and the possibility that non-essential subunits do not define alternate versions of the complex.
\end{abstract}

Keywords Yeast · Chromatin · RSC $\cdot$ Nucleosome · Mitosis

\section{Introduction}

A fundamentally different logic of gene regulation between prokaryotes and eukaryotes was previously proposed based

Communicated by M. Kupiec.

Damien Hermand

Damien.Hermand@unamur.be

1 URPHYM-GEMO, Namur Research College (NARC), The University of Namur, 5000 Namur, Belgium

2 Instituto de Biología Funcional y Genómica, Consejo Superior de Investigaciones Científicas (CSIC)/ Universidad de Salamanca, Campus Miguel de Unamuno, 37007 Salamanca, Spain on the existence of chromatin in the latter, which results in a closed, less accessible genome (Struhl 1999). Despite the fact that this view may be too simplistic-there are structural proteins associated with the DNA in prokaryotes (Anuchin et al. 2011) and eukaryotes use more repressors than anticipated (Kemmeren et al. 2014)—-the general concept still stands true (Estrada et al. 2016). Strikingly, the human genome harbours the blueprint of about 200 highly specialized cell types characterized by very different morphology, metabolism and capacities including, for example, neurons, hepatocytes or gametes. By comparison, most prokaryotes have a limited range of cellular states. Therefore, the invention of chromatin may have been pivotal for the emergence of highly differentiated cell types, most likely because the expression of specific programmes must be tightly regulated to allow diverse and sometimes antagonistic differentiated states to co-exist. For example, yeast differentiation during gametogenesis must be very strictly limited to diploid cells to avoid massive cell death resulting from haploid meiosis and recent data support that chromatin-based mechanisms play a key role in that process (van Werven et al. 2012). The understanding of how chromatin is established and how it contributes with most, if not all nuclear processes including transcription, DNA replication, DNA repair, recombination or chromosome segregation therefore constitutes an outstanding focus in current biology. In that context, an important and long-standing question is to decipher how nucleosomes, which constitute the basic unit of chromatin, are positioned genome-wide. There have been abundant debates about what dictates the position of nucleosomes with models fully relying on biophysics-the position is DNA encoded-and a model encompassing a layer of active, ATP-dependent modelling of the chromatin template. The reader is redirected to excellent reviews addressing these issues (Korber 2012; Lieleg 
et al. 2015; Struhl and Segal 2013). In the meantime, the discovery of a large set of chromatin remodelers supported the second possibility and the development of more refined nucleosome occupancy maps in various species led to the discovery of general features of nucleosome positioning along the eukaryotic genomes. Particularly, nearby the promoter, there is often a stereotypical organization just upstream of the transcription start site (TSS) with a large nucleosome-depleted region (NDR) flanked by highly positioned nucleosomes referred to as the +1 and the -1 . From these two landmarks, arrays of regularly spaced nucleosomes often extend. The remodel the structure of chromatin (RSC) complex has a specific role in the generation of NDR in budding yeast (Badis et al. 2008; Hartley and Madhani 2009; Parnell et al. 2008; Wippo et al. 2011). RSC is an abundant and essential paralog of the canonical SWI/ SNF remodeler (Cairns et al. 1996) that contains a DNAdependent ATPase (Sth1 in budding yeast, Snf21 in fission yeast) that translocates DNA and allows to shift nucleosome positions, or even completely eject nucleosomes. Importantly, RSC show compositional and functional differences between budding and fission yeasts (Monahan et al. 2008) and it was suggested that RSC is not required for NDR formation in the latter (Pointner et al. 2012). In addition, not all subunit of RSC are essential, which may indicate that subcomplexes exist and may have specialized functions. Finally, it is unclear what is the most prominent biological role of RSC, in other words which nuclear process is mainly affected upon RSC inactivation and results in lethality. Here we briefly comment on these issues by reanalysing previously published data from budding yeast and by building on our recent work in fission yeast.

\section{Defects in the RSC remodeler affects NDR formation in both budding yeast and fission yeast}

One of the early difficulties in comparing the two model yeast species (the budding yeast Saccharomyces cerevisiae, hereafter S. cerevisiae, and the fission yeast Schizosaccharomyces pombe, hereafter $S$. pombe) was the very poor annotation of the transcription start sites in S. pombe. This is an issue when plotting the average nucleosome signal obtained from MNase-Seq experiments to transcripts coordinates. Very recently, several groups (Booth et al. 2016; Eser et al. 2016; Li et al. 2015) have used different approaches to address that issue and Fig. 1a shows a comparison of the average nucleosome signal when plotted to the Pombase annotation (the reference of the community working on fission yeast) and the most recent work performed by the Lis laboratory using precision run-on $5^{\prime}$ cap sequencing (PRO-cap) (Booth et al. 2016), which corresponds to the annotation used in this manuscript. Comparing the overlay of MNase-Seq data after alignment at
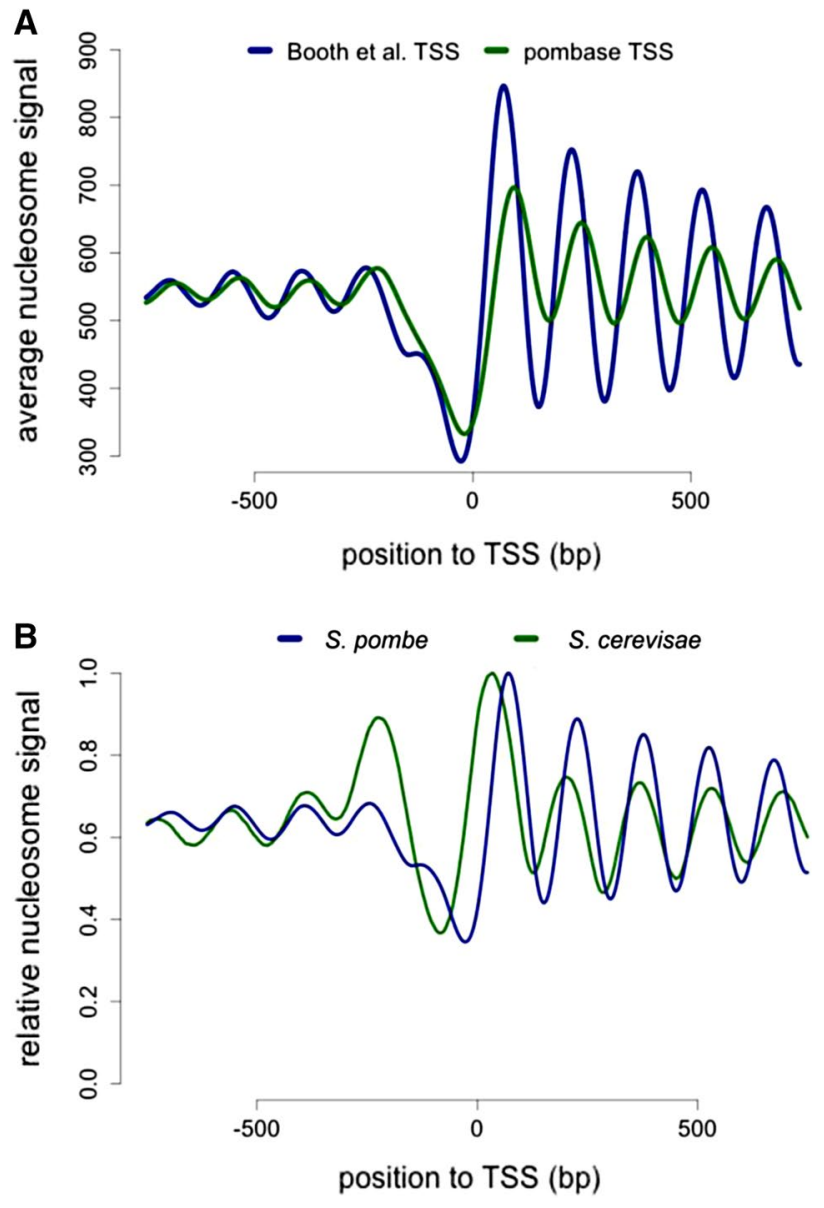

Fig. 1 Average nucleosome occupancy nearby the TSS in fission and budding yeasts. a Average nucleosome signals centered on two different fission yeast TSS annotations. b Average nucleosome signals centered on TSS in fission yeast and budding yeasts

the TSS between the two yeast species confirms previous observations from the pioneer work of the Korber laboratory in fission yeast, namely the absence of a clearly positioned -1 nucleosome and shorter nucleosome spacing in S. pombe (Lantermann et al. 2009). In addition, the +1 nucleosome is positioned further away from the TSS and there are clear, albeit weak nucleosome arrays upstream of NDR in fission yeast (Fig. 1b). Importantly, the low amplitude of the peaks and the absence of a positioned -1 nucleosome were previously shown to result from the larger variation in the size of individual NDRs in fission yeast (Soriano et al. 2013). Therefore, nucleosomal arrays emanate bidirectionally from the NDRs in fission yeast as well.

It is now well established that the ablation of RSC activity in budding yeast strongly affects the majority of NDRs (Badis et al. 2008; Hartley and Madhani 2009; Parnell et al. 2008). Recent data further confirmed that in the inactivation of RSC (resulting from switching-off the expression of $r s c 8$ ) leads to upstream and downstream nucleosomal 
arrays to shift to and eventually occlude the NDR. This work also supports the idea that phasing patterns reflect the resultant of phasing signals emanating from neighbouring NDRs (Ganguli et al. 2014). Most recently, it was reported by the Cairns laboratory that RSC and ISW1 have functional antagonism, which is supported by the fact that the gain in nucleosome occupancy in $r s c$ mutant is attenuated by the additional inactivation of ISW1 (Parnell et al. 2015).

In the fission yeast, the RSC complex was also associated with the generation of NDR in the context of heterochromatin. Indeed the deletion of $r s c l$ that encodes a non-essential subunit of RSC suppresses the requirement of the histone deacetylase (HDAC) $\mathrm{Clr} 3$ for NDR elimination (Garcia et al. 2010), which indirectly supports that RSC is responsible for acetylation-dependent NDR formation in that species. However, that study did not expand to euchromatin. Finally, it was reported that CHD1 remodelers, Hrp1 and Hrp3 are required in fission yeast to link nucleosomal arrays to most TSS (Pointner et al. 2012). In the same study, the role of RSC in nucleosome positioning was also analysed using the only conditional (thermosensitive) mutant allele available for the gene $\operatorname{snf} 21$ that encodes the catalytic subunit of RSC (Yamada et al. 2008). Unexpectedly, no effect on nucleosome positioning around TSS was obvious upon thermal inactivation of the mutant, which suggested that RSC plays no role in NDR formation in S. pombe.

Our recent work identified RSC as a key downstream effector of a cascade controlling the level of acetylation around the NDR of ste11, which encodes the master regulator of gametogenesis in $S$. pombe (Anandhakumar et al. 2013; Cassart et al. 2012; Coudreuse et al. 2010; Devos et al. 2015). Deletion of non-essential subunits of RSC or transcriptional switch-off of snf21 both impede stel1 expression and correlate with higher nucleosome occupancy at the stell NDR (Materne et al. 2015, 2016). This effect led us to analyse the genome-wide effect of both RSC mutants on nucleosome positioning using MNaseSeq, which allows us to assess the conservation of the chromatin remodelling function of the RSC complex in budding and fission yeast.

We have reanalysed the data presented in the Parnell et al. (2015) paper with the following modifications compared to the published work. First, no filtering for specific gene organization was applied. Second, the data are presented at single base pair resolution rather that within $50 \mathrm{bp}$ windows relative to the TSS as done before. This was made possible as we used the MNase-Seq data rather than the Agilent $244 \mathrm{~K}$ mircoarrays used for the main figures of the original study. Third, the TSS annotation was obtained from a different source (Ganguli et al. 2014). Figure 2a shows the profile of nucleosome occupancy ratios between the $s t h 1$ degron (a strain that allows rapid and conditional
A

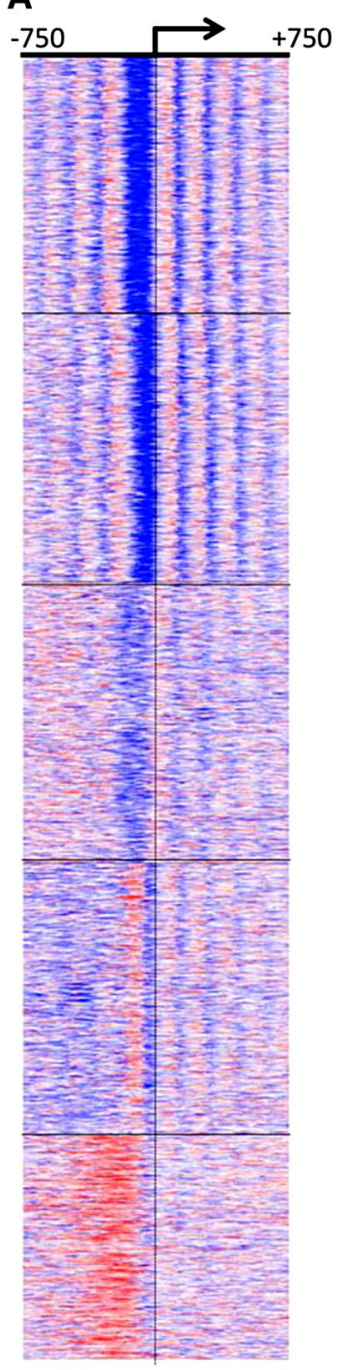

\begin{tabular}{l}
-1.5 \\
$0 \quad \log 2\left(\mathrm{NuC}_{\text {sth }}{ }^{\mathrm{deg} / \mathrm{ctr}}\right.$ ) \\
-1.5 \\
\hline
\end{tabular}

S.cerevisiae
B

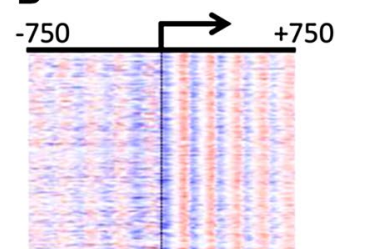

Fig. 2 Defects in the RSC remodeler affects NDR formation in both budding yeast and fission yeast. a The profile of nucleosome occupancy ratios between budding yeast sthl degron (sthl ${ }^{\text {deg }}$ ) and control strains is presented as an heatmap, where blue represents a gain in nucleosome occupancy and red represents a loss within a region ranging from $-750 \mathrm{bp}$ to $+750 \mathrm{bp}$ around the TSS at single nucleotide resolution. Rows represent genes and are organized into five groups by $k$-means clustering. b Same as in a, except that the fission yeast $r s c 1$ deletion mutant $(r s c 1 \Delta)$ and control strains are presented

degradation of the Sth1 protein RSC subunit) and control strains $\pm 750 \mathrm{bp}$ relative to the TSS. Genes were organized into clusters based on their $r s c-/ R S C+$ ratio. As reported in the published work, a leftward shift in nucleosome positions over the transcribed region is observed, confirming 
that nucleosomal arrays emanate from the NDR flanking nucleosomes. Most likely thus, NDR filling reflects encroachment by the flanking -1 and +1 nucleosomes, rather than insertion of an additional nucleosome within the NDR region as previously discussed (Ganguli et al. 2014).

We next applied identical analyses to the data obtained in a fission yeast $r s c l$ mutant. It should be noted straight away that Rsc1 is a non-essential subunit of RSC while the work done in budding yeast targeted the gene encoding the catalytic subunit. Nonetheless, the general picture obtained when $r s c l$ is absent (Fig. 2b) is reminiscent of the budding yeast data (Fig. 2a). Compared to budding yeast, the shortening of NDR is observed with a slight shift towards the TSS that recalls the shift of the position of the +1 nucleosome between budding and fission yeast (Fig. 1b). In addition, the leftward shift in nucleosome positions over the transcribed region is also obvious in most clusters. These data suggest that similarly to budding yeast, RSC also play an important conserved role in the establishment of NDRs in fission yeast in contrast to previous conclusions (Pointner et al. 2012). However, it is important to keep in mind that the previous study relies on a $t s$ allele of $\sin 21$. As rightly pointed by the authors in their manuscript, it is possible that the inactivation of the snf21-ts was not complete despite the fact that the strain has obvious phenotypes (see below), somehow masking an effect of most NDR. Supporting this possibility, we report here that while switching-off snf 21 expression using a Tet-off system eventually results in cell death on plates (Materne et al. 2015), it has a weak genome-wide effect on NDR formation when a short time point is used (Fig. 3a). This suggests that lowering RSC activity by switching-off the transcription of the $\operatorname{snf} 21$ gene (with about $35 \%$ of the $\operatorname{snf} 21$ mRNA left) may only affect the most sensitive RSC-dependent processes. These data claim for the generation of a much more efficient switch-off system that could quickly deplete the vast majority of the Snf21 protein pool in the cell.

\section{A single RSC complex containing non-essential subunits likely fulfils all RSC functions}

Interestingly, the analysis of $r s c 1$ deletion in fission yeast reveals a genome-wide effect on NDR, yet the effect appears milder than the inactivation of the catalytic subunit in S. cerevisiae (compare Fig. 2a, b). However, the shrinkage of NDR when $r s c l$ is deleted is statistically significant (one-sample Wilcoxon test $p<0.01$ ), Fig. 3b). To us, these data do not support that the non-essential subunits constitute an RSC submodule with specific functions but rather indicates that within RSC, the non-essential subunits may have a less prominent structural role than essential subunits. In line with this, all the phenotypes reported when inactivating RSC, including chromosomal segregation defects, and sensitivity to drugs, are shared to various degrees by all mutants (Monahan et al. 2008).

\section{A key role of $\mathrm{RSC}$ in mitotic chromosome condensation}

Despite the genome-wide defect observed in NDR formation (Fig. 2b), the $r s c l$ mutant has a subtle impact on steady-state transcription. Expression alteration effects in either direction were seen for only $1.4 \%$ of $S$ pombe genes in the $r s c 1$ mutant and other non-essential subunit similarly affect the transcriptome (Monahan et al. 2008). Interestingly, the third cluster in Fig. $2 b$ harbours the highest frequency of genes affected in the $r s c 1$ mutant (Fig. 3c), including stell (Materne et al. 2015, 2016). That cluster is characterized by an increase in occupancy over a broad region upstream of the TSS that may be typical of highly regulated genes relying on larger regulatory sequences, as typically seen for stell (Anandhakumar et al. 2013). Nonetheless, the main phenotypes of the $r s c 1$ mutant, and the snf21-ts mutant for that matter, are cell elongation associated with chromosomes segregation defects. Although these phenotypes may result from the reduced expression of specific genes, a recent study rather points to a direct role of RSC and nucleosome eviction in condensin loading and chromosome condensation (Toselli-Mollereau et al. 2016). A genetic screen for functional partners of conden$\sin$ in fission yeast (synthetic lethality with cut3-477 that encodes a condensin ATPase subunit) identified alleles of $\operatorname{arp} 9$ and $\operatorname{snf} 21$ that both showed high frequency of chromatin bridges in anaphase (Robellet et al. 2014). Further analyses revealed the preferred localization of condensin at, or near NDR and that increased nucleosome occupancy upon RSC downregulation is sufficient to decrease condensin binding. These data point to a prominent role of RSC is establishing the landscape of condensin binding during mitosis by establishing NDR.

In conclusion it appears that the role of RSC in NDR formation is conserved between both $S$. pombe and $S$. cerevisiae. The milder effect observed with the $r s c 1$ mutant in fission yeast may be caused by (1) the fact that this subunit is not essential and its deletion could only partially impair RSC activity; (2) the presence of an additional remodeler involved in NDR formation; (3) the fact that S. pombe lacks ISW1-type remodelers that are known to oppose the action of RSC in NDR formation in budding yeast (Parnell et al. 2008, 2015). Future work will clarify this issue. Biologically, the most prominent role of RSC may be to maintain proper chromosome segregation and may extend to kinetochore function, sister chromatid cohesion and DNA repair, in addition to its role in promoting transcription 
A

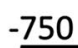

750 $+750$
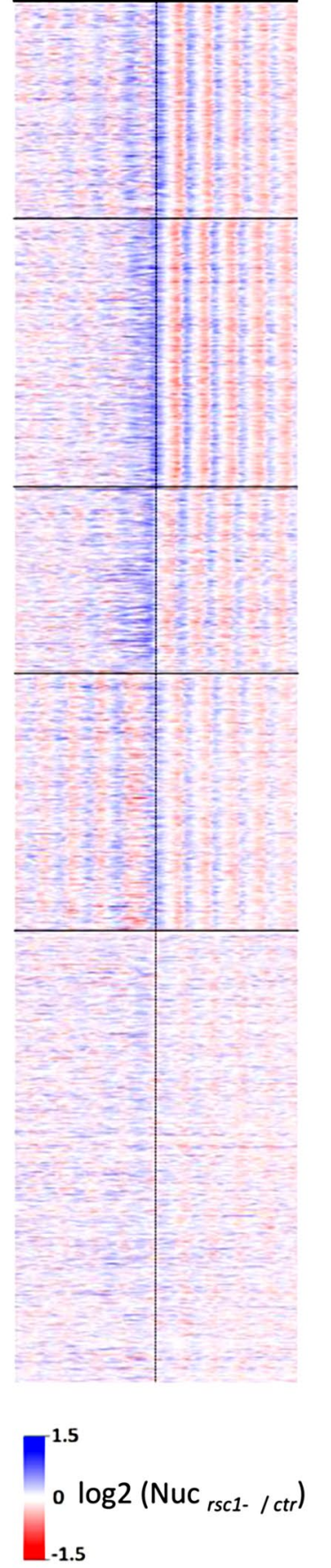

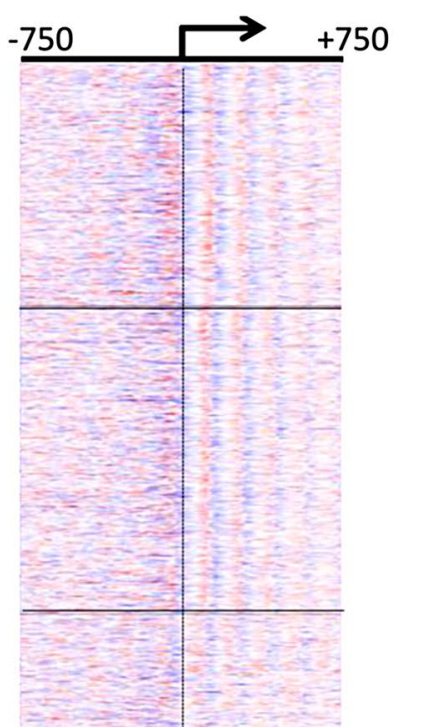

B

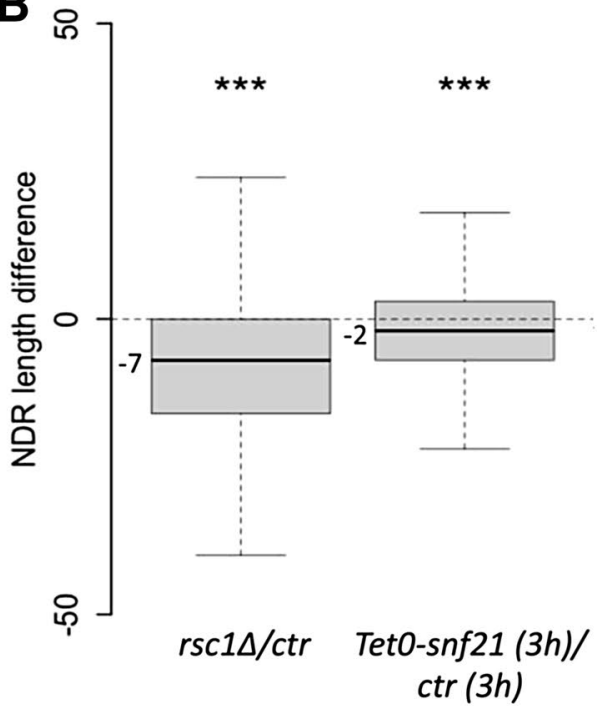

C

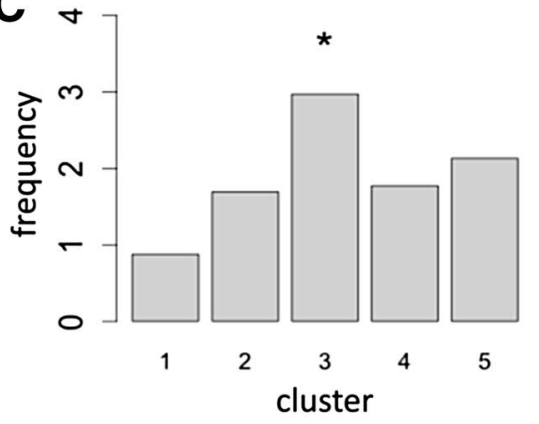

Fig. 3 Comparison of the fission yeast $r s c l$ deletion mutant and the $\operatorname{snf} 21$ switch-off strains. a The profile of nucleosome occupancy ratios between fission yeast $r s c 1$ deletion mutant $(r s c 1 \Delta)$ and control strains on the left panel (note that this panel is identical to Fig. 2b and repeated here for clarity), and between the fission yeast snf 21 switchoff mutant [tetO-snf21, $3 \mathrm{~h}$ of inhibition, (Materne et al. 2015)] and control strains on the right panel are presented as a heatmap, where blue represents a gain in nucleosome occupancy and red represents a loss within a region ranging from $-750 \mathrm{bp}$ to $+750 \mathrm{bp}$ around the TSS at single nucleotide resolution. Rows represent genes and are organized into five groups by $k$-means clustering. b Box plot representing the difference in NDR length between the $r s c 1 \Delta$ and tet $O$ snf21 strains and the corresponding wt strains. Statistical significance was calculated by a one-sample Wilcoxon test ( pval <0.01). c Frequency of genes downregulated ( $\log 2$ fold change $<-0.5$, based on (Monahan et al. 2008) in rscl $\Delta$ strain sorted by cluster. Cluster 3 includes the ste 11 gene and is enriched (Fisher's exact test $P$ value $<0.05)$ for genes whose expression is downregulated in the $r s c 1 \Delta$ strain 
(Cao et al. 1997; Hsu et al. 2003; Huang et al. 2004; Shim et al. 2007).

\section{Bioinformatic analyses}

TSS annotations for $S$. pombe were obtained from pombase (http://www.pombase.org/, ASM294v2.26) and (Booth et al. 2016). TSS annotation for S. cerevisiae was obtained from (Ganguli et al. 2014). Nucleosome genome-wide occupancy profiles at $1 \mathrm{bp}$ resolution were generated as described (Bauer and Hermand 2012; Drogat et al. 2012; Lenglez et al. 2010; Materne et al. 2015) using DANPOS for the $S$. pombe data or directly downloaded from GEO (supplementary of GSE65593) for the S. cerevisiae data (Parnell et al. 2015).

Nucleosome occupancy ratios were computed as the $\log 2$ ratio between treatment and control profiles centered at TSS for all protein coding genes. A pseudocount of +1 was added to both the numerator and the denominator to avoid division by zero. Clustering of the nucleosome occupancy ratio was made using the kmeans() function in base $\mathrm{R}$ with default parameters and $k=5$ and visualized with the heatmap.2() function from the "gplots" package.

Nucleosome positions were computed for each dataset as the local maximum of nucleosome occupancy in a $100 \mathrm{bp}$ window using the localMaximum() function from the "MassSpecWavelet" package. Promoter NDR length is computed as the distance in bases between the position of the first nucleosome before the TSS and the first nucleosome after the TSS. This metric was computed for mutants and wild type strains and subtracted accordingly to obtain the NDR length difference as in Fig. $3 b$.

\section{Data access}

The nucleosome sequencing data are available in the GEO database under the accession numbers GSE84912 ( $S$. pombe datasets) and GSE65593 (S. cerevisiae datasets).

Acknowledgments We thank Philipp Korber for critical reading of the manuscript. This work was supported by Grant BFU2014-52143-P from the Spanish Ministerio de Economía y Competitividad to FA and by Grants PR T.0012.14 to DH. CY is a FRIA Research Fellow. DH is a FNRS Senior Research Associate.

\section{Compliance with ethical standards}

Conflict of interest The authors declare that they have no conflict of interest.

Ethical approval This article does not contain any studies with human participants or animals performed by any of the authors.
Open Access This article is distributed under the terms of the Creative Commons Attribution 4.0 International License (http://creativecommons.org/licenses/by/4.0/), which permits unrestricted use, distribution, and reproduction in any medium, provided you give appropriate credit to the original author(s) and the source, provide a link to the Creative Commons license, and indicate if changes were made.

\section{References}

Anandhakumar J, Fauquenoy S, Materne P, Migeot V, Hermand D (2013) Regulation of entry into gametogenesis by Ste11: the endless game. Biochem Soc Trans 41:1673-1678

Anuchin AM, Goncharenko AV, Demidenok OI, Kaprel'iants AS (2011) Histone-like proteins of bacteria (review). Prikl Biokhim Mikrobiol 47:635-641

Badis G, Chan ET, van Bakel H, Pena-Castillo L, Tillo D, Tsui K, Carlson CD, Gossett AJ, Hasinoff MJ, Warren CL et al (2008) A library of yeast transcription factor motifs reveals a widespread function for Rsc3 in targeting nucleosome exclusion at promoters. Mol Cell 32:878-887

Bauer F, Hermand D (2012) A coordinated codon-dependent regulation of translation by Elongator. Cell Cycle 11:4524-4529

Booth GT, Wang IX, Cheung VG, Lis JT (2016) Divergence of a conserved elongation factor and transcription regulation in budding and fission yeast. Genome Res 26:799-811

Cairns BR, Lorch Y, Li Y, Zhang M, Lacomis L, Erdjument-Bromage H, Tempst P, Du J, Laurent B, Kornberg RD (1996) RSC, an essential, abundant chromatin-remodeling complex. Cell 87:1249-1260

Cao Y, Cairns BR, Kornberg RD, Laurent BC (1997) Sfh1p, a component of a novel chromatin-remodeling complex, is required for cell cycle progression. Mol Cell Biol 17:3323-3334

Cassart C, Drogat J, Migeot V, Hermand D (2012) Distinct requirement of RNA polymerase II CTD phosphorylations in budding and fission yeast. Transcription 3:231-234

Coudreuse D, van Bakel H, Dewez M, Soutourina J, Parnell T, Vandenhaute J, Cairns B, Werner M, Hermand D (2010) A gene-specific requirement of RNA polymerase II CTD phosphorylation for sexual differentiation in S. pombe. Curr Biol 20:1053-1064

Devos M, Mommaerts E, Migeot V, van Bakel H, Hermand D (2015) Fission yeast $\mathrm{Cdk} 7$ controls gene expression through both its CAK and C-terminal domain kinase activities. Mol Cell Biol 35:1480-1490

Drogat J, Migeot V, Mommaerts E, Mullier C, Dieu M, van Bakel H, Hermand D (2012) Cdk11-cyclinL controls the assembly of the RNA polymerase II mediator complex. Cell Rep 2:1068-1076

Eser P, Wachutka L, Maier KC, Demel C, Boroni M, Iyer S, Cramer P, Gagneur J (2016) Determinants of RNA metabolism in the Schizosaccharomyces pombe genome. Mol Syst Biol 12:857

Estrada J, Wong F, DePace A, Gunawardena J (2016) Information integration and energy expenditure in gene regulation. Cell 166:234-244

Ganguli D, Chereji RV, Iben JR, Cole HA, Clark DJ (2014) RSCdependent constructive and destructive interference between opposing arrays of phased nucleosomes in yeast. Genome Res 24:1637-1649

Garcia JF, Dumesic PA, Hartley PD, El-Samad H, Madhani HD (2010) Combinatorial, site-specific requirement for heterochromatic silencing factors in the elimination of nucleosome-free regions. Genes Dev 24:1758-1771

Hartley PD, Madhani HD (2009) Mechanisms that specify promoter nucleosome location and identity. Cell 137:445-458 
Hsu JM, Huang J, Meluh PB, Laurent BC (2003) The yeast RSC chromatin-remodeling complex is required for kinetochore function in chromosome segregation. Mol Cell Biol 23:3202-3215

Huang J, Hsu JM, Laurent BC (2004) The RSC nucleosome-remodeling complex is required for Cohesin's association with chromosome arms. Mol Cell 13:739-750

Kemmeren P, Sameith K, van de Pasch LA, Benschop JJ, Lenstra TL, Margaritis T, O'Duibhir E, Apweiler E, van Wageningen S, Ko CW et al (2014) Large-scale genetic perturbations reveal regulatory networks and an abundance of gene-specific repressors. Cell 157:740-752

Korber P (2012) Active nucleosome positioning beyond intrinsic biophysics is revealed by in vitro reconstitution. Biochem Soc Trans 40:377-382

Lantermann AB, Straub T, Stralfors A, Yuan GC, Ekwall K, Korber P (2009) Schizosaccharomyces pombe genome-wide nucleosome mapping reveals positioning mechanisms distinct from those of Saccharomyces cerevisiae. Nat Struct Mol Biol 17:251-257

Lenglez S, Hermand D, Decottignies A (2010) Genome-wide mapping of nuclear mitochondrial DNA sequences links DNA replication origins to chromosomal double-strand break formation in Schizosaccharomyces pombe. Genome Res 20:1250-1261

Li H, Hou J, Bai L, Hu C, Tong P, Kang Y, Zhao X, Shao Z (2015) Genome-wide analysis of core promoter structures in Schizosaccharomyces pombe with DeepCAGE. RNA Biol 12:525-537

Lieleg C, Krietenstein N, Walker M, Korber P (2015) Nucleosome positioning in yeasts: methods, maps, and mechanisms. Chromosoma 124:131-151

Materne P, Anandhakumar J, Migeot V, Soriano I, Yague-Sanz C, Hidalgo E, Mignion C, Quintales L, Antequera F, Hermand D (2015) Promoter nucleosome dynamics regulated by signalling through the CTD code. eLife 4:e09008. doi:10.7554/eLife.09008

Materne P, Vázquez E, Sánchez M, Yague-Sanz C, Anandhakumar J, Migeot V, Antequera F, Hermand D (2016) Histone H2B ubiquitylation represses gametogenesis by opposing RSC-dependent chromatin remodeling at the ste11 master regulator locus. eLife 5:e13500. doi:10.7554/eLife.3500

Monahan BJ, Villen J, Marguerat S, Bahler J, Gygi SP, Winston F (2008) Fission yeast SWI/SNF and RSC complexes show compositional and functional differences from budding yeast. Nat Struct Mol Biol 15:873-880
Parnell TJ, Huff JT, Cairns BR (2008) RSC regulates nucleosome positioning at Pol II genes and density at Pol III genes. EMBO J 27:100-110

Parnell TJ, Schlichter A, Wilson BG, Cairns BR (2015) The chromatin remodelers RSC and ISW1 display functional and chromatinbased promoter antagonism. eLife 4:e06073

Pointner J, Persson J, Prasad P, Norman-Axelsson U, Stralfors A, Khorosjutina O, Krietenstein N, Svensson JP, Ekwall K, Korber $\mathrm{P}$ (2012) CHD1 remodelers regulate nucleosome spacing in vitro and align nucleosomal arrays over gene coding regions in $S$. pombe. EMBO J 31:4388-4403

Robellet X, Fauque L, Legros P, Mollereau E, Janczarski S, Parrinello H, Desvignes JP, Thevenin M, Bernard P (2014) A genetic screen for functional partners of condensin in fission yeast. G3 (Bethesda) 4:373-381

Shim EY, Hong SJ, Oum JH, Yanez Y, Zhang Y, Lee SE (2007) RSC mobilizes nucleosomes to improve accessibility of repair machinery to the damaged chromatin. Mol Cell Biol 27:1602-1613

Soriano I, Quintales L, Antequera F (2013) Clustered regulatory elements at nucleosome-depleted regions punctuate a constant nucleosomal landscape in Schizosaccharomyces pombe. BMC Genom 14:813

Struhl K (1999) Fundamentally different logic of gene regulation in eukaryotes and prokaryotes. Cell 98:1-4

Struhl K, Segal E (2013) Determinants of nucleosome positioning. Nat Struct Mol Biol 20:267-273

Toselli-Mollereau E, Robellet X, Fauque L, Lemaire S, Schiklenk C, Klein C, Hocquet C, Legros P, N'Guyen L, Mouillard L, et al (2016) Nucleosome eviction in mitosis assists condensin loading and chromosome condensation. EMBO J 35(14):1565-1581

van Werven FJ, Neuert G, Hendrick N, Lardenois A, Buratowski S, van Oudenaarden A, Primig M, Amon A (2012) Transcription of two long noncoding RNAs mediates mating-type control of gametogenesis in budding yeast. Cell 150:1170-1181

Wippo CJ, Israel L, Watanabe S, Hochheimer A, Peterson CL, Korber $P$ (2011) The RSC chromatin remodelling enzyme has a unique role in directing the accurate positioning of nucleosomes. EMBO J 30:1277-1288

Yamada K, Hirota K, Mizuno K, Shibata T, Ohta K (2008) Essential roles of Snf21, a Swi2/Snf2 family chromatin remodeler, in fission yeast mitosis. Genes Genetic Syst 83:361-372 